\title{
Scaling of the modal response of a simply-supported rectangular plate
}

\author{
R. D. Hampton ${ }^{1}$, T. H. Li ${ }^{2}$ \& L. K. Byers ${ }^{1}$ \\ ${ }^{1}$ Civil and Mechanical Engineering Department, \\ US Military Academy, USA \\ ${ }^{2}$ Weapons \& Materials Research Directorate, \\ US Army Research Laboratory, USA
}

\begin{abstract}
Actual military transports (such as ground vehicles or ships) are often not acceptable for shock-response testing, for such reasons as cost, vehicle availability, personnel availability, or time. If testing can be conducted on scaled-down models, whether of the actual transports or of critical subsections, the results might be usable to approximate the shock response of the full-scale hardware, provided that appropriate scaling relationships can be developed. Since many military vehicles and vessels have large structural portions consisting of homogeneous flat plates, these elements serve as a logical starting point for shock-response scaling efforts. This paper develops scaling relationships for thin rectangular plates in simple support, subject to transverse point-shock loads, under the assumptions of linearity, homogeneity, and geometric similarity of the plate faces. The plates are also assumed to have the same respective modal damping ratios, for corresponding modes. It is found that simple scaling factors exist, between corresponding modal frequencies, and between corresponding damped modeshapes. Further, via suitable discretization, these factors can be used to provide simple scaling relationships between the shock response of points on a scaled-down plate and that of corresponding (scaled) points on the scaled-up plate.
\end{abstract}

Keywords: shock response, rectangular plates, modal analysis, model scaling. 


\section{Introduction}

Shock-response testing of actual, full-sized hardware is often impractical or impossible, for reasons of cost or availability. The hardware to be tested may be prohibitively expensive, limited in number, unavailable (e.g., in use), inaccessible, or even nonexistent (e.g., under development). And even should the hardware - or a suitable full-scale model - be available and released for testing, an appropriate test facility may itself be unavailable, nonexistent, or excessively time-intensive or expensive to use. In such cases it may be possible to conduct shock tests of scaled-down test models, and to scale up the results to approximate the results expected from full-sized models or the actual hardware.

The present paper describes how shock-response tests of a scaled-down model could be used to approximate anticipated test results of larger hardware, in the case of a simply-supported, homogeneous, damped rectangular plate. The larger and smaller plates must be proportional in length and width, but the thicknesses need be neither identical nor related by the same proportion. Both plates are assumed to have the same respective modal damping ratios, for corresponding modes; and the shock is assumed to be transverse, point-loaded, and ideal (Dirac-delta). Plastic deformation must be local only-restricted to the immediate region of the impact, and of negligible effect on remote-point vibratory response.

\section{Problem statement}

Referring to fig.'s 1 and 2, consider two flat, simply-supported, homogeneous rectangular plates (plate \#1: the smaller, or "test" plate; and plate \#2, the larger, or "full-scale" plate), with identical modal damping ratios for each respective pair of modes. The respective Poisson's ratios $(v)$, Young's moduli of elasticity $(E)$, and area densities $(\rho)$ need not be identical. Assume that numerical shockresponse data is available for the test plate - whether determined experimentally or otherwise. Identify its length, width, and thickness by $a_{1}, b_{1}$, and $h_{1}$, respectively. Let the full-scale plate have respective length, width, and thickness of $a_{2}, b_{2}$, and $h_{2}$, with dimensions related to those of the test plate as follows:

$$
\left[\begin{array}{lll}
a_{2} & b_{2} & h_{2}
\end{array}\right]=\left[\begin{array}{lll}
\alpha a_{1} & \alpha b_{1} & \beta h_{1}
\end{array}\right]
$$

It is desired to relate mathematically the vibratory shock responses of the two plates. In particular, let the $q^{\text {th }}$ plate $(q=1,2)$ have $k^{\text {th }}$ undamped modal frequency, $k^{\text {th }}$ damped modal frequency, and $k^{\text {th }}$ modeshape designated, respectively, by $\omega_{n, k, q}, \omega_{d, k, q}$, and $W_{k, q}$. (The subscripts $n$ and $d$ indicate "undamped" and "damped," respectively.) Then the first two objectives of this paper are as posed below: 
- Determine the relationship between undamped modal frequencies $\omega_{n, k, 1}$ and $\omega_{n, k, 2}$. (The same relationship will obtain between damped modal frequencies $\omega_{d, k, 1}$ and $\omega_{d, k, 2}$.)

- Determine the relationship between modeshapes $W_{k, 1}$ and $W_{k, 2}$.

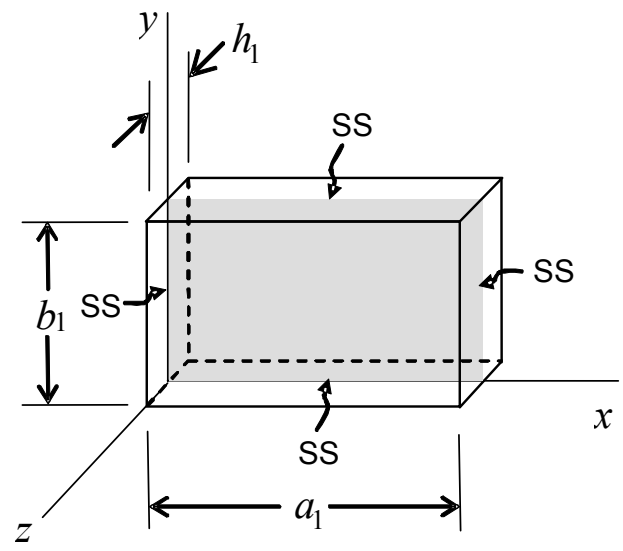

SS: Simply Supported

Figure 1: Plate \#1: Thin test plate, with indicated edges in simple support (SS).

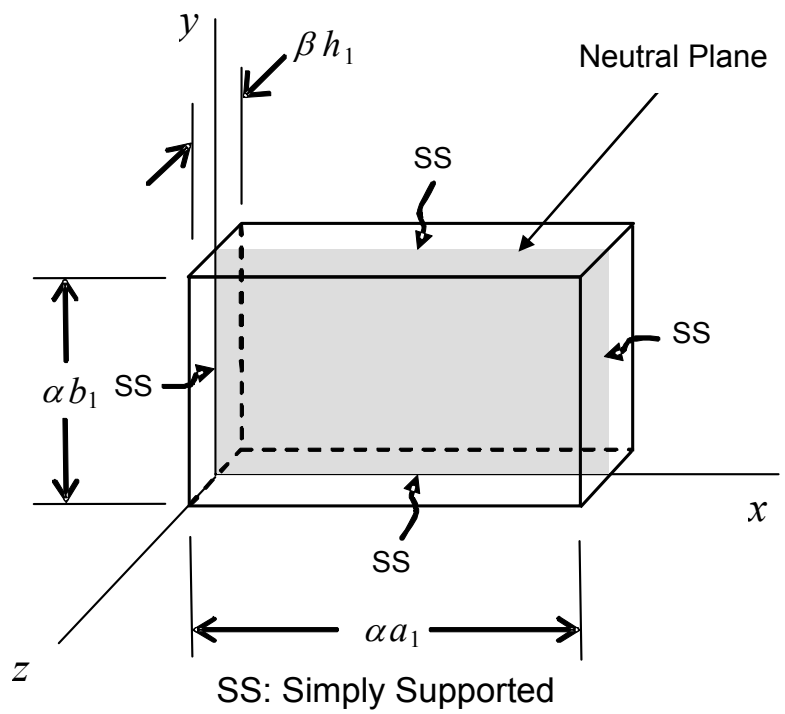

Figure 2: Plate \#2: Full-scale plate, with integral scaling as indicated. 
Refer now to fig. 3. Consider the case of the same two plates in free response to known initial conditions. The third objective is as follows:

- Under the assumption of modal damping, determine the relationship between the two plates' respective free responses $\left(w_{j, 1}\right)_{\text {free }}$ and $\left(w_{j, 2}\right)_{\text {free }}$.

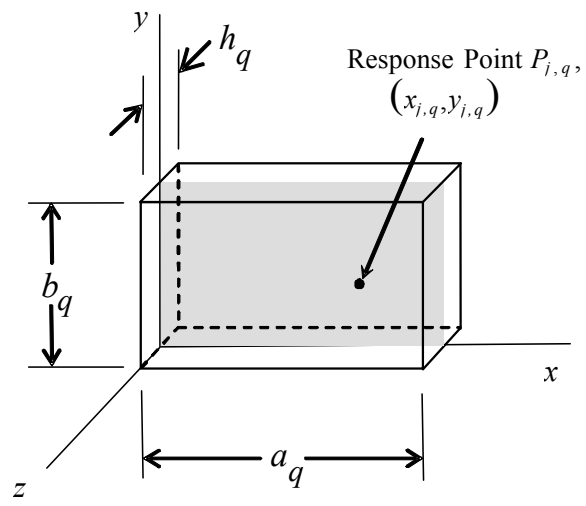

Figure 3: $\quad$ Response point for idealized plate.

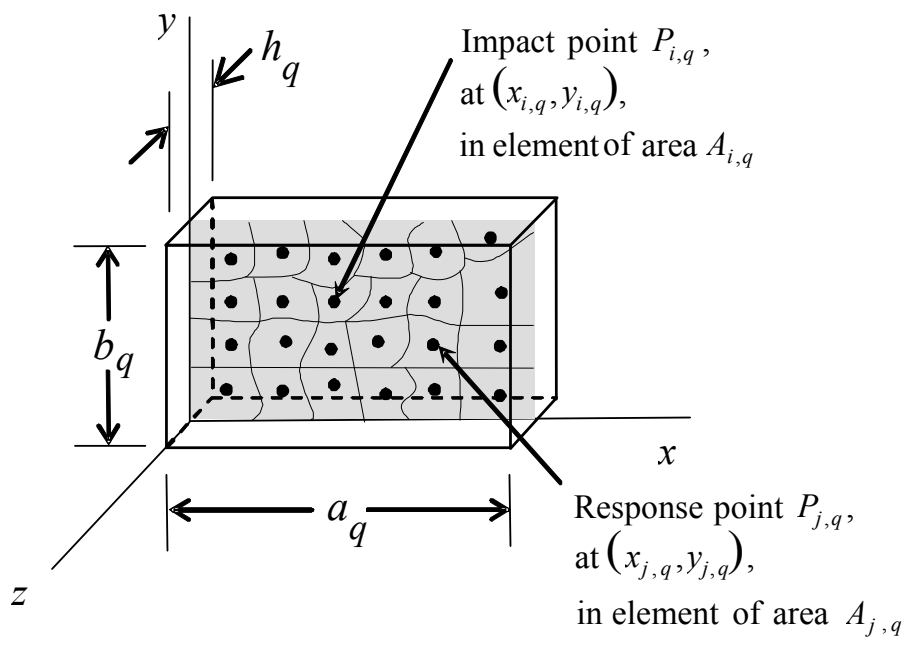

Figure 4: Discretized plate, with elements shown.

Refer now to fig. 4. For the same two plates consider the case of an ideal (Dirac-delta) impulse (having strength $\gamma_{q}$ for plate $q$ ) applied transversely at corresponding points $P_{i, q}$ on the respective plates, where the respective impact points are "proportionally" located. That is, for $P_{i, 1}$ located on the test-plate 
midplane at $\left(x_{i, 1}, y_{i, 1}\right)$ let corresponding point $P_{i, 2}$, with coordinates $\left(x_{i, 2}, y_{i, 2}\right)$ on the midplane of the full-scale plate, be located at $\left(\alpha x_{i, 1}, \alpha y_{i, 1}\right)$. Designate the respective transverse responses at corresponding points $P_{j, q}$ (also on the respective midplanes) by $\left(w_{j, q}\right)_{\text {forced }}$. If the two plates are now discretized as indicated (fig. 4), a mathematical relationship can be determined between the forced responses for the two plates. This is the final objective of the paper:

- Determine the relationship between the forced responses $\left(w_{j, 1}\right)_{\text {forced }}$ and $\left(w_{j, 2}\right)_{\text {forced }}$, for a discretized, lumped-parameter model, with the two plates subjected transversely to ideal (Dirac-delta) impulsive point-shock loads of known strengths.

The following section summarizes pertinent governing equations of the rectangular plates described above. The subsequent four sections address respectively the four objectives given above. A concluding section summarizes the results, indicates some possible applications, and suggests directions for future work.

\section{Modal response of a simply-supported rectangular plate}

Consider a flat, linear, homogenous rectangular plate (fig. 1) of length $a$ ( $x$ direction), width $b$ ( $y$-direction), uniform thickness $h$, Young's modulus $E$, Poisson's ratio $v$, and mass density $\rho$ per unit area. The plate is assumed to be simply supported around its perimeter. Assume the plate to be subject to a timevarying transverse external force intensity (i.e., per unit area of plate surface):

$$
q=q(x, y, t),
$$

with the $x$ and $y$ axes lying in the undeformed neutral plane.

The partial differential equation for the undamped plate is well-known (e.g., [1-3]):

where

$$
\rho w_{t t}+D \nabla^{4} w=q(x, y, t)
$$

$$
w=w(x, y, t)
$$

is the transverse (z-direction) displacement of the neutral surface, the flexural rigidity $D$ is defined by

$$
D=E h^{3} /\left(12\left(1-v^{2}\right)\right),
$$

the subscript notation indicates partial differentiation:

$$
w_{t t}:=\partial^{2} w / \partial t^{2},
$$

and

$$
\nabla^{4}:=\nabla^{2} \nabla^{2}
$$


is the biharmonic differential operator (the dual application of the Laplacian operator $\nabla^{2}$ ). For rectangular coordinates the Laplacian is expressed by

$$
\nabla^{2}=\partial^{2} / \partial x^{2}+\partial^{2} / \partial y^{2} .
$$

The simply supported boundary conditions are represented by

$$
w=w_{x x}+v w_{y y}=0 .
$$

An analytical, modal solution to the homogeneous form of the undamped differential equation is well known, for this plate geometry and set of boundary conditions [1-3]. In particular,

$$
w_{\text {free }}(x, y, t)=\sum_{m=1}^{\infty} \sum_{l=1}^{\infty} W_{m, l}(x, y) \eta_{m, l}(t),
$$

where the undamped natural frequency for a particular set of values for $m$ and $l$ is

$$
\omega_{n, m, l}=\pi^{2} \sqrt{D / \rho}\left[(m / a)^{2}+(l / b)^{2}\right],
$$

the associated (normalized) modeshape is

$$
W_{m, l}=\sin (m \pi x / a) \sin (l \pi y / b),
$$

and the corresponding undamped modal coordinate is

$$
\eta_{m, l}=\sin \left(\omega_{m, l} t+\phi_{m, l}\right),
$$

where the phase angles $\phi_{m, l}$ depend on the initial conditions.

If the undamped natural frequencies are arranged in increasing order, then the double indices $m, l$ can be replaced with a single index $k$, and eqns (10) through (13) can be rewritten as follows [4]:

$$
w_{\text {free }}(x, y, t)=\sum_{k=1}^{\infty} W_{k}(x, y) \eta_{k}(t)
$$

where the $k^{\text {th }}$ undamped natural frequency is

$$
\omega_{n, k}=\pi^{2} \sqrt{D / \rho}\left[(m / a)^{2}+(l / b)^{2}\right],
$$

the associated (normalized) modeshape is

$$
W_{k}(x, y)=\sin \left(\frac{m \pi x}{a}\right) \sin \left(\frac{l \pi y}{b}\right)=\sin \left(\frac{m \pi x}{a}\right) \sin \left(y \sqrt{\omega_{n, k} \sqrt{\frac{\rho}{D}}-\left(\frac{m \pi}{a}\right)^{2}}\right),
$$

and the corresponding undamped modal coordinate is

$$
\eta_{k}=\sin \left(\omega_{n, k} t+\phi_{k}\right) \text {. }
$$


With modal damping $\varsigma_{k}$, the modal coordinate becomes

$$
\eta_{d, k}=e^{-\varsigma_{k} \omega_{n, k} t} \sin \left(\omega_{n, k} t+\phi_{k}\right) .
$$

The modal free response, then, is

$$
w_{\text {free }}(x, y, t)=\sum_{k=1}^{\infty} C_{k} W_{k}(x, y) \eta_{d, k}(t),
$$

where the constants $C_{k}$, along with the phase angles $\phi_{k}$, depend on the initial conditions.

\section{Scaled modal frequencies}

From (15), the undamped natural frequencies for the $q^{\text {th }}$ plate are given by

$$
\omega_{n, k, q}=\pi^{2} \sqrt{D_{q} / \rho_{q}}\left[\left(m / a_{q}\right)^{2}+\left(l / b_{q}\right)^{2}\right] ;
$$

where, from eqn (5), $\quad D_{q}=E_{q} h_{q}^{3} /\left(12\left(1-v_{q}^{2}\right)\right)$.

In terms of their respective volumetric densities $\bar{\gamma}_{q}$, the undamped modal frequencies for the test- and full-scale-plates are, respectively,

$$
\omega_{n, k, 1}=h_{1} \pi^{2} \sqrt{E_{1} /\left(12 \bar{\gamma}_{1}\left(1-v_{1}^{2}\right)\right)}\left[\left(m / a_{1}\right)^{2}+\left(l / b_{1}\right)^{2}\right]
$$

and

$$
\omega_{n, k, 2}=\beta h_{1} \pi^{2} \sqrt{E_{2} /\left(12 \bar{\gamma}_{2}\left(1-v_{2}^{2}\right)\right)}\left[\left(m /\left(\alpha a_{1}\right)\right)^{2}+\left(l /\left(\alpha b_{1}\right)\right)^{2}\right] .
$$

It follows readily that the undamped natural frequencies are related as follows:

$$
\omega_{n, k, 2}=\left(\left(\beta / \alpha^{2}\right) \sqrt{E_{2} \bar{\gamma}_{1}\left(1-v_{1}^{2}\right) /\left(E_{1} \bar{\gamma}_{2}\left(1-v_{2}^{2}\right)\right)}\right) \omega_{n, k, 1} .
$$

For identical modal damping ratios $\varsigma_{k}$, the damped natural frequencies are similarly related:

$$
\omega_{d, k, 2}=\left(\left(\beta / \alpha^{2}\right) \sqrt{E_{2} \bar{\gamma}_{1}\left(1-v_{1}^{2}\right) /\left(E_{1} \bar{\gamma}_{2}\left(1-v_{2}^{2}\right)\right)}\right) \omega_{d, k, 1} .
$$

Defining the frequency gain, $\Gamma_{\omega}$, by

$$
\Gamma_{\omega}=\left(\beta / \alpha^{2}\right) \sqrt{E_{2} \bar{\gamma}_{1}\left(1-v_{1}^{2}\right) /\left(E_{1} \bar{\gamma}_{2}\left(1-v_{2}^{2}\right)\right)},
$$


the scaling relationships for the modal frequencies become:

$$
\omega_{n, k, 2}=\Gamma_{\omega} \omega_{n, k, 1} .
$$

and

$$
\omega_{d, k, 2}=\Gamma_{\omega} \omega_{d, k, 1}
$$

Observe that $\Gamma_{\omega}$ is constant with modal frequency (i.e., it is invariant with $k$ ).

\section{Scaled modeshapes}

From eqn (16), the undamped modeshapes for the test- and full-scale plates are, respectively,

and

$$
\begin{aligned}
& W_{k, 1}(x, y)=\sin \left(m \pi x / a_{1}\right) \sin \left(l \pi y / b_{1}\right), \\
& W_{k, 2}(x, y)=\sin \left(m \pi x / a_{2}\right) \sin \left(l \pi y / b_{2}\right)
\end{aligned}
$$

Note that eqns (29) and (30) have meaning as physical modeshapes only for coordinates describing actual points on the test and full-scale plates, respectively. That is, for eqn (29) to be physically meaningful the coordinates must be limited to values of $x$ from 0 to $a_{1}$; and of $y$, from 0 to $b_{1}$. For eqn (30), the coordinates must be limited to values of $x$ from 0 to $\alpha a_{1}$; and of $y$, from 0 to $\alpha b_{1}$. (Mathematically, of course, there are no such restrictions.) The designation $W_{k, 1}^{e x t}(x, y)$ will be used in this paper when indicating a domain for $W_{k, 1}(x, y)$ that extends beyond the test-plate boundaries.

$W_{k, 2}$ can be expressed in terms of $W_{k, 1}$ as follows. Let $\alpha$ first be expressed as the ratio of two positive integers, for reasons to be seen shortly:

$$
\alpha=\lambda / \mu \text {. }
$$

Then, substitution from eqn (31) into eqn (1) yields

$$
a_{2} / \lambda=a_{1} / \mu
$$

Accordingly, $\quad \sin \left(m \pi x /\left(a_{2} / \lambda\right)\right)=\sin \left(m \pi x /\left(a_{1} / \mu\right)\right)$;

equivalently, $\quad \sin \left(\lambda m \pi x / a_{2}\right)=\sin \left(\mu m \pi x / a_{1}\right)$.

Since $\lambda$ and $\mu$ are positive integers ([5], p. 811),

$$
\begin{gathered}
\sin \left(\lambda \frac{m \pi x}{a_{2}}\right)=\left(\begin{array}{c}
\lambda \\
1
\end{array}\right) \cos ^{\lambda-1}\left(\frac{m \pi x}{a_{2}}\right) \sin ^{1}\left(\frac{m \pi x}{a_{2}}\right)-\left(\begin{array}{l}
\lambda \\
3
\end{array}\right) \cos ^{\lambda-3}\left(\frac{m \pi x}{a_{2}}\right) \sin ^{3}\left(\frac{m \pi x}{a_{2}}\right) \\
+\left(\begin{array}{c}
\lambda \\
5
\end{array}\right) \cos ^{\lambda-5}\left(\frac{m \pi x}{a_{2}}\right) \sin ^{5}\left(\frac{m \pi x}{a_{2}}\right) \mp \cdots
\end{gathered}
$$




$$
\begin{aligned}
& =\sum_{r=0}^{\operatorname{int}((\lambda-1) / 2)}(-1)^{r}\left(\begin{array}{c}
\lambda \\
2 r+1
\end{array}\right) \cos ^{\lambda-(2 r+1)}\left(\frac{m \pi x}{a_{2}}\right) \sin ^{2 r+1}\left(\frac{m \pi x}{a_{2}}\right) \\
& =\sin \left(\frac{m \pi x}{a_{2}}\right)\left[\sum_{r=0}^{\operatorname{int}((\lambda-1) / 2)}(-1)^{r}\left(\begin{array}{c}
\lambda \\
2 r+1
\end{array}\right) \cos ^{\lambda-(2 r+1)}\left(\frac{m \pi x}{a_{2}}\right) \sin ^{2 r}\left(\frac{m \pi x}{a_{2}}\right)\right],
\end{aligned}
$$

where

$$
y=\operatorname{int}(x)
$$

represents the largest integer such that

$$
y \leq x
$$

Similarly,

$$
\begin{aligned}
\sin \left(\mu \frac{m \pi x}{a_{1}}\right) & =\left(\begin{array}{c}
\mu \\
1
\end{array}\right) \cos ^{\mu-1}\left(\frac{m \pi x}{a_{1}}\right) \sin ^{1}\left(\frac{m \pi x}{a_{1}}\right)-\left(\begin{array}{c}
\mu \\
3
\end{array}\right) \cos ^{\mu-3}\left(\frac{m \pi x}{a_{1}}\right) \sin ^{3}\left(\frac{m \pi x}{a_{1}}\right) \\
& \quad+\left(\begin{array}{c}
\mu \\
5
\end{array}\right) \cos ^{\mu-5}\left(\frac{m \pi x}{a_{1}}\right) \sin ^{5}\left(\frac{m \pi x}{a_{1}}\right) \mp \cdots \\
& =\sum_{r=0}^{\operatorname{int}((\mu-1) / 2)}(-1)^{r}\left(\begin{array}{c}
\mu \\
2 r+1
\end{array}\right) \cos ^{\mu-(2 r+1)}\left(\frac{m \pi x}{a_{1}}\right) \sin ^{2 r+1}\left(\frac{m \pi x}{a_{1}}\right) \\
= & \sin \left(\frac{m \pi x}{a_{1}}\right)\left[\sum_{r=0}^{\operatorname{int}((\mu-1) / 2)}(-1)^{r}\left(\begin{array}{c}
\mu \\
2 r+1
\end{array}\right) \cos ^{\mu-(2 r+1)}\left(\frac{m \pi x}{a_{1}}\right) \sin ^{2 r}\left(\frac{m \pi x}{a_{1}}\right)\right] .
\end{aligned}
$$

By an analogous development, one can also readily derive the following:

$$
\begin{gathered}
b_{2} / \lambda=b_{1} / \mu \\
\sin \left(\lambda m \pi x / b_{2}\right)=\sin \left(\mu m \pi x / b_{1}\right) \\
\sin \left(\lambda \frac{l \pi y}{b_{2}}\right)=\sin \left(\frac{l \pi y}{b_{2}}\right)\left[\sum_{r=0}^{\operatorname{int}((\lambda-1) / 2)}(-1)^{r}\left(\begin{array}{c}
\lambda \\
2 r+1
\end{array}\right) \cos ^{\mu-(2 r+1)}\left(\frac{l \pi y}{b_{2}}\right) \sin ^{2 r}\left(\frac{l \pi y}{b_{2}}\right)\right] \\
\sin \left(\mu \frac{l \pi y}{b_{1}}\right)=\sin \left(\frac{l \pi y}{b_{1}}\right)\left[\sum_{r=0}^{\operatorname{int}((\mu-1) / 2)}(-1)^{r}\left(\begin{array}{c}
\mu \\
2 r+1
\end{array}\right) \cos ^{\mu-(2 r+1)}\left(\frac{l \pi y}{b_{1}}\right) \sin ^{2 r}\left(\frac{l \pi y}{b_{1}}\right)\right] .
\end{gathered}
$$

Define now the following:

$$
\begin{gathered}
\Gamma_{\lambda x, k}(x)=\left[\sum_{r=0}^{\operatorname{int}((\lambda-1) / 2)}(-1)^{r}\left(\begin{array}{c}
\lambda \\
2 r+1
\end{array}\right) \cos ^{\lambda-(2 r+1)}\left(\frac{m \pi x}{a_{2}}\right) \sin ^{2 r}\left(\frac{m \pi x}{a_{2}}\right)\right], \\
\Gamma_{\mu x, k}(x)=\left[\sum_{r=0}^{\operatorname{int}((\mu-1) / 2)}(-1)^{r}\left(\begin{array}{c}
\mu \\
2 r+1
\end{array}\right) \cos ^{\mu-(2 r+1)}\left(\frac{m \pi x}{a_{1}}\right) \sin ^{2 r}\left(\frac{m \pi x}{a_{1}}\right)\right],
\end{gathered}
$$




$$
\begin{aligned}
\Gamma_{\lambda y, k}(y) & =\left[\sum_{r=0}^{\operatorname{int}((\lambda-1) / 2)}(-1)^{r}\left(\begin{array}{c}
\lambda \\
2 r+1
\end{array}\right) \cos ^{\mu-(2 r+1)}\left(\frac{l \pi y}{b_{2}}\right) \sin ^{2 r}\left(\frac{l \pi y}{b_{2}}\right)\right], \\
\text { and } \quad \Gamma_{\mu y, k}(y) & =\left[\sum_{r=0}^{\operatorname{int}((\mu-1) / 2)}(-1)^{r}\left(\begin{array}{c}
\mu \\
2 r+1
\end{array}\right) \cos ^{\mu-(2 r+1)}\left(\frac{l \pi y}{b_{1}}\right) \sin ^{2 r}\left(\frac{l \pi y}{b_{1}}\right)\right] .
\end{aligned}
$$

Then eqns (37), (42), (45), and (46) can be written, respectively, as:

$$
\begin{aligned}
\sin \left(\lambda m \pi x / a_{2}\right) & =\Gamma_{\lambda x, k}(x) \sin \left(m \pi x / a_{2}\right), \\
\sin \left(\mu m \pi x / a_{1}\right) & =\Gamma_{\mu x, k}(x) \sin \left(m \pi x / a_{1}\right), \\
\sin \left(\lambda l \pi y / b_{2}\right) & =\Gamma_{\lambda y, k}(y) \sin \left(l \pi y / b_{2}\right), \\
\sin \left(\mu l \pi y / b_{1}\right) & =\Gamma_{\mu y, k}(y) \sin \left(l \pi y / b_{1}\right)
\end{aligned}
$$

Since, by eqns (34) and (44), the four expressions in eqns (51) and (52) are mutually equivalent,

$$
\sin \left(\frac{m \pi x}{a_{2}}\right)=\frac{\Gamma_{\mu x, k}(x)}{\Gamma_{\lambda x, k}(x)} \sin \left(\frac{m \pi x}{a_{1}}\right)
$$

Similarly, the expressions in eqns (53) and (54) are mutually equivalent, so that

$$
\sin \left(l \pi y / b_{2}\right)=\left(\Gamma_{\mu y, k}(y) / \Gamma_{\lambda y, k}(y)\right) \sin \left(l \pi y / b_{1}\right)
$$

Substitution from eqns (55) and (56) into eqn (30) leads directly to the desired scaling relationship for the modeshapes:

$$
W_{k, 2}(x, y)=\Gamma_{W, k}(x, y) W_{k, 1}^{e x t}(x, y),
$$

where the $k^{\text {th }}$ modeshape gain, $\Gamma_{w, k}$, is

$$
\Gamma_{W, k}(x, y)=\left(\Gamma_{\mu x, k}(x) \Gamma_{\mu y, k}(y)\right) \div\left(\Gamma_{\lambda x, k}(x) \Gamma_{\lambda y, k}(y)\right) .
$$

\section{Scaled free response}

It is now possible to write a full expression of a scaled free response. For a testplate free response described by

$$
w_{1, \text { free }}(x, y, t)=\sum_{k=1}^{\infty} C_{k, 1} W_{k, 1}(x, y) e^{-\varsigma_{k} \omega_{n, k, 1} t} \sin \left(\omega_{d, k, 1} t+\phi_{k, 1}\right),
$$


the corresponding free response of the full-scale plate is

$$
w_{2, \text { free }}(x, y, t)=\sum_{k=1}^{\infty} C_{k, 2} \Gamma_{W, k}(x, y) W_{k, 1}^{e x t}(x, y) e^{-\varsigma_{k} \Gamma_{\omega} \omega_{n, k, 1} t} \sin \left(\Gamma_{\omega} \omega_{d, k, 1} t+\phi_{k, 2}\right),
$$

where the modal gains (scaling factors) $\Gamma_{\omega}$ and $\Gamma_{W, k}(x, y)$ are as defined by eqns (26) and (58), supported by eqns (47) through (50). The coefficients $C_{k, q}$ and the phase angles $\phi_{k, q}$ depend on the initial conditions, as noted previously.

\section{Scaled point response, for discretized plate with point loading}

For the test plate, the modal response at point $P_{j, 1}$ to an ideal impulse of strength $\gamma_{1}$ at point $P_{i, 1}$ is

$$
\left(w_{j, 1}\right)_{\text {forced }}=\frac{\gamma_{1}}{m_{i, 1}} \sum_{k=1}^{p} \frac{u_{j, k, 1} v_{k, i, 1}}{\omega_{d, k, 1}} e^{-\varsigma_{k} \omega_{n, k, 1} t} \sin \left(\omega_{d, k, 1} t+\phi_{k, 1}\right),
$$

where $u_{j, k, 1}$ is the $(j, k)$ element of the normalized modeshape matrix $U_{1}$ for the discretized plate [4], and $v_{k, i, 1}$ is the $(k, i)$ element of that matrix's pseudoinverse, $U_{1}^{+}$. (As described in [4], the normalization is accomplished by making each discretized modeshape of unit length.) Similarly, for the full-scale plate, the modal response at point $P_{j, 2}$ to an ideal impulse of strength $\gamma_{2}$ at point $P_{i, 2}$ is

$$
\left(w_{j, 2}\right)_{\text {forced }}=\frac{\gamma_{2}}{m_{i, 2}} \sum_{k=1}^{p} \frac{u_{j, k, 2} v_{k, i, 2}}{\omega_{d, k, 2}} e^{-\varsigma_{k} \omega_{n, k, 2} t} \sin \left(\omega_{d, k, 2} t+\phi_{k, 2}\right),
$$

where $u_{j, k, 2}$ and $v_{k, i, 2}$ are analogously defined.

From eqn (30),

$$
W_{k, 2}(\alpha x, \alpha y)=\sin \left(m \pi \alpha x / a_{2}\right) \sin \left(l \pi \alpha y / b_{2}\right) .
$$

Substitution from (1) into (63) leads directly to

$$
W_{k, 2}(\alpha x, \alpha y)=\sin \left(m \pi x / a_{1}\right) \sin \left(l \pi y / b_{1}\right),
$$

so that, applying eqn (29) to eqn (64),

$$
W_{k, 2}(\alpha x, \alpha y)=W_{k, 1}(x, y) \text {. }
$$

(Notice the difference between eqn (57) and the above equation. The former relates the scaled-plate modeshape at any point to the extended modeshape of the 
test-plate for the same point; the latter equates the scaled-plate modeshape at a scaled point to the test-plate modeshape at the corresponding unscaled point.)

By using the scaling relationships of eqns (28) and (66), and with corresponding points on the two plates proportionally located (as defined in section 2), one can now modify eqn (62) to express the modal forced response of the full-scale plate in terms of the modal parameters of the test plate. Key substitutions are made as follows:

$$
\begin{aligned}
& u_{j, k, 2}=u_{j, k, 1}, \\
& v_{j, k, 2}=v_{j, k, 1},
\end{aligned}
$$

and

$$
m_{i, 2}=m_{i, 1}\left(\alpha^{2} \beta \rho_{2} / \rho_{1}\right)
$$

Accordingly, $\left(w_{j, 2}\right)_{\text {forced }}=\frac{\gamma_{2} \Gamma_{R}}{m_{i, 1}} \sum_{k=1}^{p}\left(\frac{u_{j, k, 1} v_{k, i, 1}}{\omega_{d, k, 1}}\right) e^{-\varsigma_{k} \Gamma_{\omega} \omega_{n, k, 1} t} \sin \left(\Gamma_{\omega} \omega_{d, k, 1} t+\phi_{k, 2}\right)$,

where the parameter $\Gamma_{R}$ is a shock-response gain with definition

$$
\Gamma_{R}=\rho_{1} /\left(\alpha^{2} \beta \rho_{2} \Gamma_{\omega}\right)
$$

Equivalently, $\quad \Gamma_{R}=\rho_{1} /\left(\rho_{2} \beta^{2}\right) \sqrt{E_{1} \bar{\gamma}_{2}\left(1-v_{2}^{2}\right) /\left(E_{2} \bar{\gamma}_{1}\left(1-v_{1}^{2}\right)\right)}$

For proportionally located impact and response points, eqn (60) becomes

$$
w_{2, \text { free }}(\alpha x, \alpha y, t)=\sum_{k=1}^{\infty} C_{k, 2} W_{k, 1}(x, y) e^{-\varsigma_{k} \Gamma_{\omega} \omega_{n, k, 1} t} \sin \left(\Gamma_{\omega} \omega_{d, k, 1} t+\phi_{k, 2}\right),
$$

By limiting the free response to $p$ modes, and summing the respective free and forced responses (for the test plate, from eqns (59) and (61); for the fullscale plate, from eqns (72) and (69)), the total responses of the discretized plates can now be expressed. For the test plate, the total response at point $P_{j, 1}$ to an ideal impulse of strength $\gamma_{1}$ at point $P_{i, 1}$ is:

$$
\begin{gathered}
w_{1}\left(x_{j, 1}, y_{j, 1}, t\right)=w_{1, \text { free }}\left(x_{j, 1}, y_{j, 1}, t\right)+w_{1, \text { forced }}\left(x_{j, 1}, y_{j, 1}, t\right) \\
=\sum_{k=1}^{p} C_{k, 1} W_{k, 1}\left(x_{j, 1}, y_{j, 1}\right) e^{-\varsigma_{k} \omega_{n, k, 1} t} \sin \left(\omega_{d, k, 1} t+\phi_{k, 1}\right) \\
+\left(\gamma_{1} / m_{i, 1}\right) \sum_{k=1}^{p}\left(u_{j, k, 1} v_{k, i, 1} / \omega_{d, k, 1}\right) e^{-\varsigma_{k} \omega_{n, k, 1} t} \sin \left(\omega_{d, k, 1} t+\phi_{k, 1}\right) \\
=\sum_{k=1}^{p}\left(C_{k, 1} W_{k, 1}\left(x_{j, 1}, y_{j, 1}\right)+\left(\gamma_{1} / m_{i, 1}\right)\left(u_{j, k, 1} v_{k, i, 1} / \omega_{d, k, 1}\right)\right) e^{-\varsigma_{k} \omega_{n, k, 1} t} \sin \left(\omega_{d, k, 1} t+\phi_{k, 1}\right) .
\end{gathered}
$$


Similarly, for the full-scale plate, the total response at point $P_{j, 2}$, located at $\left(x_{j, 2}=\alpha x_{j, 1}, y_{j, 2}=\alpha y_{j, 1}\right)$ to an ideal impulse of strength $\gamma_{2}$ at point $P_{i, 2}$, located at $\left(x_{i, 2}=\alpha x_{i, 1}, y_{i, 2}=\alpha y_{i, 1}\right)$ is:

$$
\begin{gathered}
w_{2}\left(x_{j, 2}, y_{j, 2}, t\right)=w_{2, \text { free }}\left(x_{j, 2}, y_{j, 2}, t\right)+w_{2, \text { forced }}\left(x_{j, 2}, y_{j, 2}, t\right) \\
=\sum_{k=1}^{p} C_{k, 2} W_{k, 2}\left(x_{j, 2}, y_{j, 2}\right) e^{-\varsigma_{k} \omega_{n, k, 2} t} \sin \left(\omega_{d, k, 2} t+\phi_{k, 2}\right) \\
+\left(\gamma_{2} / m_{i, 2}\right) \sum_{k=1}^{p}\left(u_{j, k, 2} v_{k, i, 2} / \omega_{d, k, 2}\right) e^{-\varsigma_{k} \omega_{n, k, 2} t} \sin \left(\omega_{d, k, 2} t+\phi_{k, 2}\right) \\
=\sum_{k=1}^{p}\left(C_{k, 2} W_{k, 2}\left(x_{j, 2}, y_{j, 2}\right)+\left(\frac{\gamma_{2}}{m_{i, 2}}\right)\left(\frac{u_{j, k, 2} v_{k, i, 2}}{\omega_{d, k, 2}}\right)\right) e^{-\varsigma_{k} \omega_{n, k, 2} t} \sin \left(\omega_{d, k, 2} t+\phi_{k, 2}\right) .
\end{gathered}
$$

In terms of test-plate parameters, the total response of the full-scale plate is:

$$
\begin{gathered}
w_{2}\left(x_{j, 2}, y_{j, 2}, t\right)=w_{2, \text { free }}\left(x_{j, 2}, y_{j, 2}, t\right)+w_{2, \text { forced }}\left(x_{j, 2}, y_{j, 2}, t\right) \\
=\sum_{k=1}^{p} C_{k, 2} W_{k, 1}\left(x_{j, 1}, y_{j, 1}\right) e^{-\varsigma_{k} \Gamma_{\omega} \omega_{n, k, 1} t} \sin \left(\Gamma_{\omega} \omega_{d, k, 1} t+\phi_{k, 2}\right) \\
+\left(\gamma_{2} \Gamma_{R} / m_{i, 1}\right) \sum_{k=1}^{p}\left(u_{j, k, 1} v_{k, i, 1} / \omega_{d, k, 1}\right) e^{-\zeta_{k} \Gamma_{\omega} \omega_{n, k, 1} t} \sin \left(\Gamma_{\omega} \omega_{d, k, 1} t+\phi_{k, 2}\right) .
\end{gathered}
$$

Upon collecting terms, one obtains

$$
w_{2}\left(x_{j, 2}, y_{j, 2}, t\right)=\sum_{k=1}^{p}\left(C_{k, 2} W_{k, 1}\left(x_{j, 1}, y_{j, 1}\right)+\left(\frac{\gamma_{2} \Gamma_{R}}{m_{i, 1}}\right)\left(\frac{u_{j, k, 1} v_{k, i, 1}}{\omega_{d, k, 1}}\right)\right) e^{-\zeta_{k} \Gamma_{\omega} \omega_{n, k, 1} t} \sin \left(\Gamma_{\omega} \omega_{d, k, 1} t+\phi_{k, 2}\right)
$$

For the common case of zero initial conditions, eqns (75) and (81) reduce, respectively, to:

$$
w_{\mathrm{i}}\left(x_{j, 1}, y_{j, 1}, t\right)=\left(\gamma_{1} / m_{i, 1}\right) \sum_{k=1}^{p}\left(u_{j, k, 1} v_{k, i, 1} / \omega_{d, k, 1}\right) e^{-\varsigma_{k} \omega_{n, k, 1} t} \sin \left(\omega_{d, k, 1} t\right)
$$

$$
\text { and } \quad w_{2}\left(x_{j, 2}, y_{j, 2}, t\right)=\left(\gamma_{2} \Gamma_{R} / m_{i, 1}\right) \sum_{k=1}^{p}\left(u_{j, k, 1} v_{k, i, 1} / \omega_{d, k, 1}\right) e^{-\zeta_{k} \Gamma_{\omega} \omega_{n, k, 1} t} \sin \left(\Gamma_{\omega} \omega_{d, k, 1} t\right) \text {. }
$$

The frequency gain $\Gamma_{\omega}$ (eqn (26)) and the shock-response gain $\Gamma_{R}$ (eqns (70) and (71)) are known functions of plate geometric and material parameters. 


\section{Initial verification of scaling equations}

In order to verify frequency-gain equation (26) two linear finite-element models were developed, one scaled geometrically relative to the other; otherwise the two plates are identical. Each model describes an isotropic, homogeneous, aluminum test-plate. For each the plate material is 6061-T6 aluminum, with Young's modulus $69 \mathrm{GPa}$, mass density $2700 \mathrm{~kg} / \mathrm{m}^{3}$, Poisson's ratio 0.33 , and modal damping ratio 0.02 (all modes). The unscaled plate is simply supported along each of its four edges, with dimensions $1.0 \mathrm{~m} \times 0.75 \mathrm{~m} \times 25 \mathrm{~mm}$; the scaled plate is twice as long, twice as wide, and one-and-a-half times as thick. (I.e., $(\alpha, \beta)=(2,1.5)$-refer to eqn (1)). Each FEA model comprises uniform rectangular (parallelepiped) elements: 50 element divisions along the length ( $x$ direction, measured in the neutral plane from the lower left corner), and 38 along the width ( $y$-direction, measured correspondingly). The modal frequencies determined by FEA were found to scale according to the analytical frequency gain $\Gamma_{\omega}$ determined above, eqn (26) - for detailed results, see table 1 . The shock-response gain, $\Gamma_{R}$ (eqns (70) and (71)), remains to be verified.

Table 1: Comparison of frequency scaling between FEA and analytical models.

\begin{tabular}{|c|c|c|c|}
\hline \multicolumn{4}{|c|}{$\begin{array}{l}\text { Modal Frequencies }(\mathrm{Hz}) \text { Comparison: First } 20 \text { Modes } \\
\text { scaled by alpha }=2 \text {, beta }=1.5\end{array}$} \\
\hline Original & Scaled by ANSYS & Scaled analytically & Difference (\%) \\
\hline 168.61 & 63.235 & 63.228 & 0.01150 \\
\hline 350.65 & 131.52 & 131.50 & 0.02254 \\
\hline 492.28 & 184.64 & 184.60 & 0.02071 \\
\hline 654.03 & 245.34 & 245.26 & 0.03094 \\
\hline 674.12 & 252.91 & 252.80 & 0.04574 \\
\hline 977.17 & 366.69 & 366.44 & 0.06959 \\
\hline 1031.6 & 386.98 & 386.85 & 0.03180 \\
\hline 1078.7 & 404.67 & 404.51 & 0.03916 \\
\hline 1213.1 & 455.21 & 454.92 & 0.06377 \\
\hline 1401.4 & 525.98 & 525.51 & 0.08917 \\
\hline 1515.6 & 568.94 & 568.36 & 0.10202 \\
\hline 1624.6 & 609.51 & 609.21 & 0.04821 \\
\hline 1786.4 & 670.22 & 669.91 & 0.04610 \\
\hline 1939.1 & 728.15 & 727.15 & 0.13825 \\
\hline 1946.6 & 730.76 & 729.99 & 0.10576 \\
\hline 1967.5 & 738.41 & 737.81 & 0.08125 \\
\hline 2269.3 & 852.07 & 850.97 & 0.12876 \\
\hline 2291.6 & 859.85 & 859.35 & 0.05833 \\
\hline 2483.4 & 932.84 & 931.26 & 0.16991 \\
\hline 2612.9 & 981.04 & 979.85 & 0.12081 \\
\hline
\end{tabular}

\section{Application}

In order to use these results one could first determine analytically the damped and undamped modal frequencies, the modeshapes, and the lumped masses for the discretized test plate, via the procedure of [4]. Next, one could determine the frequency- and shock-response gains, via the definitions found above in eqn 
(26) and either eqn (70) or eqn (71). One could then use eqn (83) to evaluate the shock response at the desired response point for a scaled plate (whether scaled up or down); no finite element analysis would be required-nor would any experimental testing, except as needed for verification.

\section{Conclusion}

This paper has developed equations by which the shock response of one simplysupported rectangular plate can be related to that of a scaled plate also under simple support, when the scaling is such that the faces of the two plates are geometrically similar. The plates are assumed to be linear; and the shock, to be ideal (Dirac-delta) transverse point-shock loading. The shock impulses may be of different strengths; and the plates may have different Young's moduli, Poisson's ratios, and volumetric densities. Modal damping is assumed, with identical damping ratios for corresponding modes. The analysis led to analytical expressions for the modal-frequency-, modeshape-, and shock-response gains.

Future work includes verifying further the above scaling relationships, finding corresponding relationships for plates with different boundary conditions, and determining situations in which these simple assumptions and geometries could be useful for real equipment and more realistic shock scenarios. Verifying these relationships could begin with finite-element analysis, but determining the degree of applicability to real equipment will likely require shock tests to physical hardware.

\section{Acknowledgements}

The authors are grateful to the Army Research Laboratory (via the U.S. Military Academy's Mathematical Sciences Center of Excellence), and to the U.S. Military Academy's Department of Civil and Mechanical Engineering, for their funding of this work.

\section{References}

[1] Leissa, A.W., Vibration of Plates, NASA SP-160, 1969.

[2] Leissa, A.W., "The Free Vibration of Rectangular Plates," Journal of Sound and Vibration, 31(3), pp. 257-293, 1973.

[3] Harris, C.M. \& Piersol, A.G., editors, Harris' Shock and Vibration Handbook, Fifth Edition, McGraw-Hill, New York, 2002, chap. 23.

[4] Hampton, R.D., Li, T.H., \& Nygren, K.P., "Analytical Shock Response of a Transversely Point-Loaded Linear Rectangular Plate," Proceedings of the $14^{\text {th }}$ ARL/USMA Technical Symposium, November 2006.

[5] Korn, G.A. \& Korn, T.M., Mathematical Handbook for Scientists and Engineers, Second Edition, McGraw-Hill, New York, 1968. 